\title{
PERIOD AND INDEX OF GENUS ONE CURVES OVER NUMBER FIELDS
}

\author{
SHAHED SHARIF
}

\begin{abstract}
The period of a curve is the smallest positive degree of Galoisinvariant divisor classes. The index is the smallest positive degree of rational divisors. We construct examples of genus one curves with prescribed period and index over certain number fields.
\end{abstract}

\section{INTRODUCTION}

Let $X$ be a nonsingular, projective, geometrically integral curve over a field $K$. Define the index $I(X)$ to be the greatest common divisor of $[L: K]$, where $L$ varies over finite algebraic field extensions for which $X(L) \neq \emptyset$. In particular, if $X$ has a rational point over $K$ already, the index is 1 . Define the period $P(X)$ of $X$ to be smallest positive degree amongst Galois-invariant divisor classes on $X$. That is, if $\bar{K}$ is an algebraic closure for $K$, then we consider divisor classes on $\bar{X}:=X \times \bar{K}$ which are fixed by the Galois action $\operatorname{Gal}(\bar{K} / K)$. We have $P(X) \mid I(X)$ since the Galois orbit of any $x \in X(L)$ yields an invariant divisor class. However, the two need not be equal: take the example of a conic without rational points, say (the projective curve given by) $x^{2}+y^{2}=-1$ over $\mathbb{R}$. Clearly, the index is 2 , but the class of a single point is Galois invariant.

In [10, Lichtenbaum showed that

Theorem 1.1. For $X / K$ as above, if the genus, period, and index of $X$ are $g, P$, and $I$ respectively, then $P|I| 2 P^{2}, I \mid(2 g-2)$, and if either $(2 g-2) / I$ or $P$ is even, then $I \mid P^{2}$.

However, over local fields and $C_{1}$ fields, the above divisibility conditions are not sharp. For example, for genus 1 curves over local fields, the period and index are always equal [10, and over $\mathbb{R}$, the index of any curve must divide 2 . One may then ask what triples $(g, P, I)$ actually occur as the genus, period, and index of a curve over a fixed $K$. Over local fields of characteristic not 2 , the problem is solved in [18. We consider the $g=1$ case over number fields and prove a full converse:

Theorem 1.2. Let $K$ be a number field and $E$ an elliptic curve over $K$. Let $\ell$ and $n$ be positive integers such that $\ell \mid n$. Then there exists a genus 1 curve $X$ with Jacobian $E$ for which $P(X)=n$ and $I(X)=n \ell$.

Note that for $g=1,(2 g-2) / I$ is always even; therefore by Theorem 1.1 we have $I \mid P^{2}$. Over any number field, then, we have covered every possible period and index for genus 1 curves.

Date: March 26, 2009.

The author would like to thank Bjorn Poonen and Pete Clark for very helpful conversations. 
The significance of period and index lies in computations of Tate-Shafarevich and Brauer groups. Grothendieck [7, §4 et seq.] is the canonical source for these computations. Poonen and Stoll [16] constructed Jacobians of curves with TateShafarevich group of nonsquare order; their construction depended on indices of the curve over various completions being maximal, and in particular not equal to the period. Gonzalez-Avilés [6] showed that given a (suitably nice) curve $X$ over a global field $K$, under certain hypotheses the finiteness of the Brauer group of a model for $X$ is equivalent to the finiteness of the Tate-Shafarevich group of the Jacobian of $X$. The relationship between the sizes of these groups is then computable, and depends on the indices and periods of $X$ both over $K$ and over the completions of $K$. Liu, Lorenzini, and Raynaud extended this result ([11, Theorem 4.3] and [12]). They showed that for $K$ the function field of a curve over a finite field, and assuming that for some prime $\ell$ the $\ell$-part of $\operatorname{Br} X$ is finite, or that the Tate-Shafarevich group of the Jacobian of $X$ is finite, we have $\operatorname{Br} X$ is finite and has square order.

In a forthcoming paper [5] the author with Pete Clark uses a result similar to Theorem 1.2 to show that if $E$ is an elliptic curve over a number field $K, p$ is a prime and $N$ any integer, then there exists a $p$-extension $L / K$ such that $\# \amalg(E / L)[p] \geq$ $N$; that is, the order of the $p$-torsion of the Tate-Shafarevich group is unbounded over $p$-extensions. The proof depends on the existence over $K$ of genus 1 curves with period $p$ and index $p^{2}$; indeed, the results of that paper imply that over any number field, there are genus 1 curves with period $N$ and index $N^{2}$.

For other results exhibiting curves with various periods and indices, see Cassels [1], Lang-Tate [8], Stein [20, O'Neil [14, and Clark [2, 3] and [4]. The strongest previous results in this direction are Clark's, who proved that when $E[p] \subset E(K)$ for $p$ prime, there are principal homogeneous spaces for $E$ with period $p$ and index $p^{2}$, and that there are curves of every index over every number field.

Recently, Stix 21 has shown that if $X$ is a curve over $\mathbb{Q}$ and there is a section for the canonical map $\pi_{1}(X) \rightarrow \pi_{1}(\mathbb{Q})$, then the period and index of $X$ are equal.

The proof of Theorem 1.2 depends fundamentally on O'Neil's obstruction map, which was introduced in [14. The necessary background on the obstruction map, as well as other basic facts, will be covered in $\S 2$. The map allows one, over an appropriate division field, to compute the index using a Hilbert symbol. In $\S 3$, we use the Chebotarev density theorem and a Hilbert symbol computation to prove Theorem 1.2 over the chosen division field. To pull the result back to the base field, we use the corestriction map in Galois cohomology, which transforms our curve over the division field to one over the base field; this is done in $\S 4$. There is some extra subtlety in the case that $n$ is even, which we dispense with in $\S 5$.

\section{Preliminary Results}

2.1. Basic properties of period and index. One can alternatively define the index to be the smallest positive degree of a divisor on $X$ over $K$. If $x \in X(\bar{K})$, the Galois orbit of $x$ written as a divisor $\sum\left({ }^{\sigma} x\right)$ furnishes a rational divisor of positive degree. Any prime divisor (of $\operatorname{Div} X$, not $\operatorname{Div} \bar{X}$ ) is necessarily of this form. This shows the equivalence of the two definitions.

There is also an alternate definition of the period. Choose any $x \in X(\bar{K})$. The cocycle $\sigma \mapsto{ }^{\sigma} x-x$ furnishes a cohomology class in $\xi \in \mathrm{H}^{1}(K, J)$, where $J$ is 
the Jacobian variety of $X$. Let $\operatorname{Pic}_{X / K}^{1}$ be the connected component of the Picard scheme of $X$ representing degree 1 invertible sheaves; it is a principal homogeneous space for $J=\operatorname{Pic}_{X / K}^{0}$. As such, there is a class in $\mathrm{H}^{1}(K, J)$ representing $\operatorname{Pic}_{X / K}^{1}$, and in fact one sees that this class is $\xi$. Similarly, $n \xi$ represents $\operatorname{Pic}_{X / K}^{n}$. We know that $\operatorname{Pic}_{X / K}^{n}$ is a trivial principal homogeneous space for $J$ precisely when it possesses a $K$-point. Such a point occurs when there is a Galois-invariant element of $\operatorname{Pic}^{n} \bar{X}$. Thus we may define the period as the order of $\xi$.

Note that when $X$ is a genus one curve, $X$ itself equals $\operatorname{Pic}_{X / K}^{1}$, and $\xi$ represents $X$ as an element of $\mathrm{H}^{1}(K, E)$, where $E$ is the Jacobian of $X$. We say a field $L$ splits $X$ if $X(L) \neq \emptyset$. For $X$ a genus 1 curve represented by $\xi \in \mathrm{H}^{1}(K, E), L$ splits $X$ if and only if $\xi$ lies in the kernel of the restriction map $\mathrm{H}^{1}(K, E) \rightarrow \mathrm{H}^{1}(L, E)$.

We now reduce the proof of Theorem 1.2 to the case where $n$ (and hence $\ell$ ) is a prime power.

Lemma 2.1. Let $P_{1}, P_{2}, I_{1}$, and $I_{2}$ be positive integers such that the $P_{i}$ are relatively prime and $P_{i}\left|I_{i}\right| P_{i}^{2}$. Let $E$ be an elliptic curve over a field $K$. If there are genus one curves $X_{1}$ and $X_{2}$ over $K$ with Jacobian $E$ such that $X_{i}$ has period $P_{i}$ and index $I_{i}$, then there is also a curve $X$ with Jacobian $E$ having period $P_{1} P_{2}$ and index $I_{1} I_{2}$.

Proof. Let $\xi_{i} \in \mathrm{H}^{1}(K, E)$ represent $X_{i}$. I claim that $\xi:=\xi_{1}+\xi_{2}$ has period $P_{1} P_{2}$ and index $I_{1} I_{2}$.

Since the period is the order of $\xi$ in $\mathrm{H}^{1}(K, E)$, the first part of the claim is obvious. Now suppose that $\xi$ has index $I$. If $L$ is a finite extension of $K$ which splits $\xi$, then $\xi$ lies in the kernel of the restriction map res : $\mathrm{H}^{1}(K, E) \rightarrow \mathrm{H}^{1}(L, E)$. Since the orders of the $\xi_{i}$ are relatively prime and res is a homomorphism, $L$ splits both of the $\xi_{i}$ as well. Therefore $I_{1} I_{2}$ divides $I$. On the other hand, any field which splits both of the $\xi_{i}$ splits $\xi$ as well. In particular, we can choose fields of the form $L_{1} \cdot L_{2}$ where each $L_{i}$ splits $\xi_{i}$. Varying over all such choices, one sees that $I \mid I_{1} I_{2}$.

2.2. O'Neil's obstruction map. Our main tool for computing the index is O'Neil's obstruction map. In order to define it, we first construct a theta group. Let $E[n]$ be the $n$-torsion of the elliptic curve $E$. Let $\mathscr{L} \in \operatorname{Pic} E$ be the invertible sheaf corresponding to the divisor $n O$, where $O$ is the identity of $E$. Our theta group $\mathscr{G}(n)$ is defined to be the set of pairs $(\varphi, \tau)$, where $\tau: E \rightarrow E$ is a translation and $\varphi$ is an isomorphism $\tau^{*} \mathscr{L} \rightarrow \mathscr{L}$. It is a group via

$$
(\varphi, \tau) \cdot\left(\varphi^{\prime}, \tau^{\prime}\right)=\left(\tau^{\prime *}(\varphi) \circ \varphi^{\prime}, \tau \circ \tau^{\prime}\right) .
$$

Note that for our choice of $\mathscr{L}, \tau$ must be translation by an element of $E[n]$. We obtain an exact sequence of $K$-group schemes

$$
0 \rightarrow \mathbb{G}_{m} \rightarrow \mathscr{G}(n) \rightarrow E[n] \rightarrow 0
$$

The subgroup $\mathbb{G}_{m}$ corresponds to the set $(a, i d)$, where $i d$ is the identity map and $a$ denotes multiplication by the constant $a$. The quotient map to $E[n]$ sends $(\varphi, \tau)$ to $\tau(O)$. Note that the group $\mathscr{G}(n)$ is nonabelian.

We consider the $\bar{K}$-points of the group schemes and take (nonabelian) Galois cohomology. There is a coboundary map

$$
\text { Ob }: \mathrm{H}^{1}(K, E[n]) \rightarrow \mathrm{H}^{2}\left(K, \mathbb{G}_{m}\right)=\mathrm{Br} K .
$$


This is the obstruction map. We recall the definition of the coboundary: for $\xi$ a cocycle representing a class in $\mathrm{H}^{1}(K, E[n])$, we lift $\xi$ to a 1-cochain $c$ with values in $\mathscr{G}(n)$. Then the obstruction map is the class of the 2-cocycle

$$
(\delta c)(\sigma, \tau)=c(\sigma)(\sigma c(\tau)) c(\sigma \tau)^{-1}
$$

with values in $\mathbb{G}_{m}$.

Please note that $\mathrm{Ob}$ is not a homomorphism! The exact sequence (2.1) is a central extension, so by the main result of Zarhin's paper [22, it is a quadratic map; that is, $\mathrm{Ob}(\xi)=b(\xi, \xi)$ for some bilinear map $b$.

Recall the Kummer sequence for $E$ gives rise to the exact sequence in Galois cohomology

$$
0 \rightarrow E(K) / n E(K) \rightarrow \mathrm{H}^{1}(K, E[n]) \rightarrow \mathrm{H}^{1}(K, E)[n] \rightarrow 0 .
$$

Thus, if $X$ has period dividing $n$, there exist (usually many) $\xi \in \mathrm{H}^{1}(K, E[n]$ ) representing it; that is, the image of $\xi$ in $\mathrm{H}^{1}(K, E)[n]$ represents $X$ in the usual sense.

In 14, O'Neil showed

Proposition 2.2. The image of the obstruction map lies in $(\mathrm{Br} K)[n]$. If $\mathrm{Ob}(\xi)$ has order $\ell$, then the index of the curve $X$ represented by $\xi$ divides n $\ell$. If $X$ has index $n$, then there is a class $\xi$ representing $X$ such that $\mathrm{Ob}(\xi)=0$.

The idea is that $\mathrm{H}^{1}(K, E[n])$ classifies principal homogeneous spaces $X$ for $E$ over $K$ along with a choice of Galois-invariant degree $n$ invertible sheaf $\mathscr{L} \in(\operatorname{Pic} \bar{X})^{G}$, up to isomorphism over $K$. Specifically, any cocycle in the class of $\xi$ gives rise to a $\bar{K}$-isomorphism $\varphi: E \rightarrow X$. Then $\mathscr{L}=\mathscr{L}(n \varphi(O))$. One sees that $\Gamma(\bar{X}, \mathscr{L}) / \bar{K}^{\times}$ forms a twist of $\mathbb{P}^{n-1}$ over $K$ - that is, a Brauer-Severi variety. The obstruction map takes the pair $(X, \mathscr{L})$ to the class of this Brauer-Severi variety in $\operatorname{Br} K$. From this characterization, the proposition is easy: If $X$ has index $n$, then there is a rational divisor $D$ of degree $n$. The class $\xi$ representing $(X, \mathscr{L}(D))$ satisfies $\mathrm{Ob}(\xi)=0$. If $\mathrm{Ob}(\xi)$ has order $\ell$ and $\xi$ represents the pair $(X, \mathscr{L})$, then the Brauer-Severi variety arising from $\mathscr{L}$ is split by an extension of degree $\ell$. Then the tensor product $\mathscr{L}^{\ell}$ is a class of degree $n \ell$ containing a rational divisor. See [14] for more details.

One would hope that the converse held: if $X$ has index $n \ell$, then there ought to be $\xi$ representing $X$ such that $\mathrm{Ob}(\xi)$ has order $\ell$. However, this is not known to be true. We will use a trick in proving the main theorem to construct $X$ for which this does hold.

Let $\delta$ be the composition $E(K) \rightarrow E(K) / n E(K) \rightarrow \mathrm{H}^{1}(K, E[n])$, where the latter map is given by the map in $(2.2)$. For $x \in E(K)$ and $X$ a principal homogeneous space for $E$ over $K$, let $T(x, X)$ denote the Tate pairing of $X$ with the image of $x$ in $E(K) / n E(K)$. Then

Proposition 2.3. $\mathrm{Ob}(\xi+\delta x)=\mathrm{Ob}(\xi)+T(x, X)$.

Proof. See [14, §5].

O'Neil's introduction of the obstruction map is useful because in many cases it may be computed using a Hilbert symbol, which we now define. Let $K$ be a field of characteristic not dividing $n$ which contains $\mu_{n}$, the $n$th roots of unity. Let $a, b \in K^{\times} / K^{\times n}$. By Kummer theory, we know that $K^{\times} / K^{\times n}=\mathrm{H}^{1}\left(K, \mu_{n}\right)$. The cup product gives a map

$$
K^{\times} / K^{\times n} \times K^{\times} / K^{\times n} \rightarrow \mathrm{H}^{2}\left(K, \mu_{n} \otimes \mu_{n}\right) .
$$


Fix a primitive $n$th root of unity $\zeta$. Define an isomorphism $\mu_{n} \otimes \mu_{n} \rightarrow \mu_{n}$ by $\zeta^{i} \otimes \zeta^{j} \mapsto \zeta^{i j}$, which induces an isomorphism $\mathrm{H}^{2}\left(K, \mu_{n} \otimes \mu_{n}\right) \rightarrow \mathrm{H}^{2}\left(K, \mu_{n}\right)$. Using the fact that $\mathrm{H}^{2}\left(K, \mu_{n}\right)=(\mathrm{Br} K)[n]$, we see that the composition gives a pairing

$$
\begin{aligned}
K^{\times} / K^{\times n} \times K^{\times} / K^{\times n} & \rightarrow(\mathrm{Br} K)[n] \\
(a, b) & \mapsto\langle a, b\rangle
\end{aligned}
$$

which we call the Hilbert symbol. If we let $w=(a, b)$, then we will alternatively write $\langle w\rangle$ for $\langle a, b\rangle$. Since the cup product is bilinear and skew-symmetric, so is the Hilbert symbol.

Note that the Hilbert symbol depends on $n$ and $\zeta$. Frequently we will abuse notation and define the Hilbert symbol as a map $\left(K^{\times}\right)^{2} \rightarrow \mathrm{Br} K$, by composing with the obvious quotient map.

We return to the obstruction map. Assume that the $n$-torsion of $E$ is rational over $K$. By the theory of the Weil pairing, $\mu_{n} \subset K$. Let $\zeta$ be the previously chosen primitive $n$th root of unity. Fix a basis $(S, T)$ for $E[n]$ such that $e(S, T)=\zeta$, where $e$ is the Weil pairing. The choice of basis, and our fixed generator $\zeta$ of $\mu_{n}$, yields an isomorphism of (trivial) Galois-modules $E[n] \cong \mu_{n} \times \mu_{n}$, and we have an isomorphism

$$
\kappa: \mathrm{H}^{1}(K, E[n]) \rightarrow K^{\times} / K^{\times n} \times K^{\times} / K^{\times n} .
$$

Proposition 2.4. Let $\xi \in \mathrm{H}^{1}(K, E[n])$. If $n$ is odd or $E[2 n] \subset E(K)$, then $\mathrm{Ob}(\xi)=$ $\langle\kappa(\xi)\rangle$. If $n$ is even, then $2 \mathrm{Ob}(\xi)=2\langle\kappa(\xi)\rangle$.

Proof. For the case that $n$ is odd, see [14, Prop. 3.4] and [15]. A proof in the even case, including an explicit computation of $\mathrm{Ob}(\xi)-\langle\kappa(\xi)\rangle$, can be found in [5], $\S 2]$.

Assume from now on that $K$ is a number field. If $v$ is a place of $K$, write $K_{v}$ for the completion of $K$ at $v$. In order to use the Hilbert symbol, we will reduce to the local case using the fact that, if $K$ is a global field, $\operatorname{Br} K=\oplus_{v} \operatorname{Br} K_{v}$. That is, in order to compute $\langle a, b\rangle$, it suffices to compute $\langle a, b\rangle_{v}$, where the latter symbol is computed in $K_{v}$, and $a, b$ are considered as elements of $K_{v}$.

(More generally, for any $K$-group scheme $M$ and integer $q$, we have a natural localization map

$$
\mathrm{H}^{q}(K, M) \rightarrow \mathrm{H}^{q}\left(K_{v}, M\right)
$$

in étale cohomology. We will denote this map by adding the subscript $v$; e.g. $\xi \mapsto \xi_{v}$.)

If $v$ is a place of $K$, we also use $v$ to denote a fixed corresponding valuation.

Lemma 2.5. Let $K_{v}$ be a nonarchimedean local field such that $v(n)=0$. Let $\pi$ be a uniformizing parameter and $u, u^{\prime}$ units; that is, $v(u)=v\left(u^{\prime}\right)=0$. Let $\mathbb{F}$ be the residue field of $K_{v}$.

(1) $\left\langle u, u^{\prime}\right\rangle_{v}=0$.

(2) The order of $\langle u, \pi\rangle_{v}$ equals the order of the image of $u$ in $\mathbb{F}^{\times} / \mathbb{F}^{\times n}$.

Proof. According to [17, Ch. XIV], $\langle a, b\rangle_{v}=0$ if and only if $b$ is a norm from the extension $K_{v}\left(a^{1 / n}\right) / K_{v}$. The extension $K_{v}\left(u^{1 / n}\right)$ is unramified with degree, say, $d$, which by Hensel's Lemma also equals the order of $u$ in $\mathbb{F}^{\times} / \mathbb{F}^{\times n}$. According to local class field theory, the norm from an unramified extension of degree $d$ is precisely the set of elements $x$ such that $v(x)$ is a multiple of $d v(\pi)$. The result follows immediately for the first claim, and from bilinearity for the second claim. 
We also have the following:

Proposition 2.6. For $a, b \in K^{\times}, \sum_{v}\langle a, b\rangle_{v}=0$.

Here, we use the fact that $\operatorname{Br} K_{v}$ is canonically isomorphic to $\mathbb{Q} / \mathbb{Z}, \frac{1}{2} \mathbb{Z} / \mathbb{Z}$, or 0 via the invariant map so that the sum makes sense. For the remainder of this paper, we identify $\operatorname{Br} K_{v}$ with the appropriate subgroup of $\mathbb{Q} / \mathbb{Z}$. Note that the sum is finite: there are finitely many archimedean primes, and for all but finitely many nonarchimedean $v, v(a)=v(b)=v(n)=0$, so that by Lemma 2.5. $\langle a, b\rangle_{v}=0$.

Proof. This follows from the fact that $\operatorname{Br} K$ is isomorphic to the set of $\left(t_{v}\right), t_{v} \in$ $\operatorname{Br} K_{v}$, such that almost all $t_{v}=0$ and $\sum t_{v}=0$; and also that cup products, and hence the Hilbert symbol, commute with localization.

Since the obstruction map does not necessarily give a sharp bound on index, we will use obstruction maps of different levels. We define $\mathrm{Ob}_{n}$ to be the corresponding obstruction map $\mathrm{H}^{1}(K, E[n]) \rightarrow \mathrm{Br} K$.

Proposition 2.7. Let $n$ and $m$ be positive integers. The following diagrams commute:

(1)

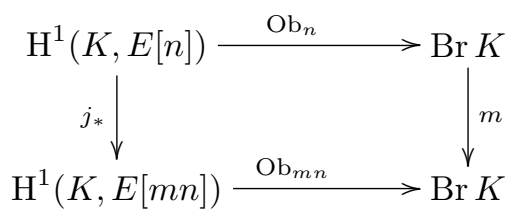

where $j_{*}$ is induced by the canonical inclusion $j: E[n] \rightarrow E[m n]$, and $m$ is muliplication by $m$.

$(2)$

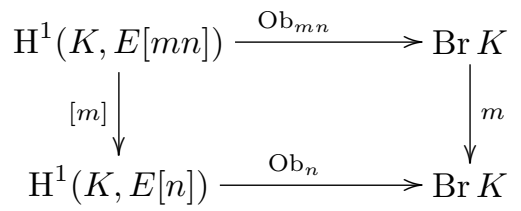

where $[m]$ is the map induced by the multiplication by $m$ map $E[m n] \rightarrow$ $E[n]$.

(3)

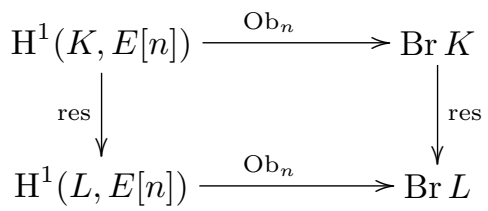

where $L / K$ is a field extension and res is the restriction map.

Proof. Let $\xi \in \mathrm{H}^{1}(K, E[n])$ represent the pair $(X, \mathscr{L})$, where $\mathscr{L}$ is the divisor class of $n(x)$ for some $x \in X(\bar{K})$. Then $j_{*}(\xi)$ represents $\left(X, \mathscr{L}^{m}\right)$. The map which takes elements of $(\operatorname{Pic} \bar{X})^{G}$ to the class of the associated Brauer-Severi 
variety is a homomorphism; indeed, it arises from the Leray spectral sequence $\mathrm{H}^{p}\left(K, \mathrm{H}^{q}\left(\bar{X}, \mathbb{G}_{m}\right)\right) \Rightarrow \mathrm{H}^{p+q}\left(X, \mathbb{G}_{m}\right)$, which yields the exact sequence

$$
0 \rightarrow \operatorname{Pic} X \rightarrow \mathrm{H}^{0}(K, \operatorname{Pic} \bar{X}) \rightarrow \mathrm{H}^{2}\left(K, \mathbb{G}_{m}\right) .
$$

The last map sends an invertible sheaf to the class of its associated Brauer-Severi variety; see for example [9. The first part of the proposition follows.

For the second part, Mumford showed [13, p. 309-310] that multiplication by $m$ extends to a homomorphism on theta groups $\mathscr{G}(m n) \rightarrow \mathscr{G}(n)$. The restriction of this homomorphism to $\mathbb{G}_{m}$ is also multiplication by $m$. Taking the long cohomology sequence associated to (2.1), we obtain the commutative diagram.

The third part is obvious, since res $\xi$ represents the same pair $(X, \mathscr{L})$ as $\xi$ does, but over $L$.

Similar to $\mathrm{Ob}_{n}$, we define $\delta_{n}$ to be the composition $E(K) \rightarrow E(K) / n E(K) \rightarrow$ $\mathrm{H}^{1}(K, E[n])$, where the second map in the composition is the coboundary coming from the Kummer sequence for $E$.

\section{The CASE OF RATIONAL TORSION}

By Lemma 2.1. we may assume that $n$ is a prime power $p^{r}$; this assumption holds for the remainder of the paper. In this section, we will prove the theorem under the additional assumption that $E[n] \subset E(K)$ in the case that $p$ is odd, and $E[2 n] \subset E(K)$ if $p=2$.

3.1. Choosing a pair of primes. Our first step is to find a pair of distinct nonarchimedean primes $v, v^{\prime}$ satisfying certain splitting conditions. (We use primes and places interchangeably.) We state the conditions below, after which we show that there exist infinitely many pairs $v, v^{\prime}$ satisfying the conditions. The proof is essentially repeated use of the Chebotarev density theorem. Let $S$ be the union of the primes $w$ of $K$ such that $E$ has bad reduction at $w$, archimedean primes, and primes dividing $n$. The conditions are

A1. The primes $v, v^{\prime}$ are principal with totally positive generators $\pi$ and $\pi^{\prime}$ respectively.

A2. Let $E(K)$ embed in $E\left(K_{v}\right)$ in the usual manner. Then $E(K)$ lies in $n E\left(K_{v}\right)$.

A3. For each $w \in S, \pi$ and $\pi^{\prime}$ lie in $K_{w}^{\times n}$.

A4. The order of the image of $\pi^{\prime}$ in $K_{v}^{\times} / K_{v}^{\times n}$ is exactly $n$.

Lemma 3.1. There exist infinitely many pairs of distinct primes $v, v^{\prime}$ satisfying conditions $A 1-A 4$.

Proof. Condition A1 is equivalent to $v$ and $v^{\prime}$ splitting completely in the Hilbert class field of $K$.

Condition A2 is the same as requiring $v$ to split completely in $K\left([n]^{-1} E(K)\right)$; that is, the field obtained by adjoining to $K$ all $x \in E(\bar{K})$ such that $[n] x \in E(K)$. By [19, p.194], $K\left([n]^{-1} E(K)\right)$ is a finite abelian extension which is unramified outside $S$.

Let $\mathfrak{m}$ be the modulus given by the product of $n^{2}$ and all primes where $E$ has bad reduction. Let $K_{\mathfrak{m}}$ be the ray class field for $K$ with modulus $\mathfrak{m}$. Then condition A3 holds if $v$ and $v^{\prime}$ split completely in $K_{\mathfrak{m}}$; for in that case, the Frobenius for $v$ (say) is trivial and, by class field theory, $v$ has a generator $\pi$ which is congruent to 1 (mod $\mathfrak{m})$. Then Hensel's Lemma implies A3. 
We now choose $v$ to be any prime which splits completely in the compositum of the three fields named above. Next we tackle A4.

By abuse of notation, let $v$ be a valuation corresponding to the prime $v$. Let $\alpha \in K$ be any element such that $v(\alpha)=0$ and whose image in $K_{v}^{\times} / K_{v}^{\times n}$ has order $n$; since $\pi \equiv 1(\bmod \mathfrak{m})$, and $n \mid \mathfrak{m}$, there exists such $\alpha$ in $K_{v}$. But $K$ is dense in $K_{v}$, so we may find such $\alpha$ in $K$. Let $F^{\prime}$ be the ray class field with modulus $v$. By class field theory, the Galois group $\operatorname{Gal}\left(F^{\prime} / K\right)$ is isomorphic to the class group with modulus $v$. In particular, if $v^{\prime}$ and $(\alpha)$ lie in the same class in this class group, then $v^{\prime}$ has a generator $\pi^{\prime}$ which is congruent to $\alpha(\bmod v)$, and hence satisfies A4.

Let $F$ be the compositum of $K_{\mathrm{m}}$ and $K\left([n]^{-1} E(K)\right)$. We see that $F^{\prime}$ is unramified outside $v$, while $F$ is unramified at $v$. Hence $F \cap F^{\prime}$ lies in the Hilbert class field of $K$. To satisfy A1-A3, we wish $v^{\prime}$ to split completely in $F$, while to satisfy A4, we wish $v^{\prime}$ to lie in the class of $(\alpha)$ in the appropriate ray class group. These conditions are compatible since they both imply that $v^{\prime}$ splits completely in the Hilbert class field of $K$. Thus we may apply the Chebotarev density theorem to find infinitely many such $v^{\prime}$ and $\pi^{\prime}$.

3.2. Construction of curve. As mentioned earlier, a choice of ordered basis $(S, T)$ for $E[n]$ with $e(S, T)=\zeta$ yields an isomorphism

$$
\kappa: \mathrm{H}^{1}(K, E[n]) \rightarrow K^{\times} / K^{\times n} \times K^{\times} / K^{\times n} .
$$

Choose $\xi \in \mathrm{H}^{1}(K, E[n])$ such that $\kappa(\xi)=\left(\pi, \pi^{\prime n / \ell}\right)$. Let $X$ be the corresponding principal homogeneous space for $E$.

Proposition 3.2. The curve $X$ has period $n$ and index $n \ell$.

Proof. For any positive integer $m$, we have $\kappa(m \xi)=\left(\pi^{m}, \pi^{\prime m n / \ell}\right)$. Let $v$ be the place of $K$ lying over $(\pi)$, and suppose $m<n$. If the curve associated to $m \xi$ is a trivial principal homogeneous space, then there exists $x \in E(K)$ such that $\kappa(\delta x+m \xi)=(1,1)$, where we recall that $\delta$ is the composition

$$
E(K) \rightarrow E(K) / n E(K) \rightarrow \mathrm{H}^{1}(K, E[n]) .
$$

But by condition A2, $x$ lies in $n E\left(K_{v}\right)$. Hence $(\delta x)_{v}=0$. Therefore for any choice of $x, \kappa(\delta x+m \xi)_{v}=\left(\pi^{m}, \cdot\right)$, and so $m \xi$ yields a trivial principal homogeneous space if and only if $n \mid m$. Therefore the period of $X$ is $n$.

By Proposition 2.4 $\mathrm{Ob}(\xi)=\left\langle\pi, \pi^{\prime n / \ell}\right\rangle$. We compute the Hilbert symbol locally. For places $w$ satisfying $w(n)>0$, condition A3 shows that

$$
\left\langle\pi, \pi^{\prime n / \ell}\right\rangle_{w}=0
$$

Let $v^{\prime}$ be the place corresponding to $\pi^{\prime}$. For $w \neq v, v^{\prime}$ and $w(n)=0, \pi$ and $\pi^{\prime}$ are both units in $K_{w}$, and so by Lemma 2.5 the local Hilbert symbol is zero. By Proposition 2.6 the order of the Hilbert symbol at $v$ equals that at $v^{\prime}$, so we need only consider $v$. Combining condition A4 with Lemma 2.5. we see that $\left\langle\pi, \pi^{\prime n / \ell}\right\rangle$ has order $\ell$. Hence $\mathrm{Ob}(\xi)$ has order $\ell$, and the index of $X$ divides $n \ell$.

Let $\ell^{\prime}$ strictly divide $\ell$, and let $j_{*}$ be the canonical map

$$
\mathrm{H}^{1}(K, E[n]) \rightarrow \mathrm{H}^{1}\left(K, E\left[n \ell^{\prime}\right]\right) .
$$

If $X$ had index $n \ell^{\prime}$, then by Lemma 2.2 there would exist $x$ in $E(K)$ such that

$$
\mathrm{Ob}_{n \ell^{\prime}}\left(\delta_{n \ell^{\prime}} x+j_{*}(\xi)\right)=0
$$


According to Proposition 2.3 , the above equals

$$
\mathrm{Ob}_{n \ell^{\prime}}\left(j_{*}(\xi)\right)+T(x, X) \text {. }
$$

By condition A2, $x \in n E\left(K_{v}\right)$ and hence $T(x, X)_{v}=0$. But by Proposition 2.7. $\mathrm{Ob}_{n \ell^{\prime}} j_{*}(\xi)=\ell^{\prime} \mathrm{Ob}_{n} \xi \neq 0$ if $\ell^{\prime}<\ell$. Therefore the index of $X$ is precisely $n \ell$.

\section{Proof of Main Theorem, odd $n$}

For now, we assume $n$ is odd and lift our hypothesis on $K$ (namely $E[n] \subset E(K)$ ). Over $L:=K(E[n])$, we may use the arguments of the previous section to construct a cohomology class $\xi \in \mathrm{H}^{1}(L, E[n])$ with the desired properties. Let cores be the corestriction map

$$
\text { cores : } \mathrm{H}^{1}(L, E[n]) \rightarrow \mathrm{H}^{1}(K, E[n]) .
$$

We will show that cores $\xi$ represents a curve over $K$ with period $n$ and index $n \ell$. In order to compute the index, we will need to base extend back to $L$; in other words, we'll compute res o cores $\xi$, where res is the restriction map

$$
\text { res : } \mathrm{H}^{1}(K, E[n]) \rightarrow \mathrm{H}^{1}(L, E[n]) \text {. }
$$

4.1. Pairs of primes. As in the previous section, we wish to choose principal primes of $L,(\pi)$ and $\left(\pi^{\prime}\right)$, such that analogues to conditions A1-A4 hold, along with a new condition. For any place $w$ of $L$, let $w_{K}$ denote the place of $K$ lying below $w$. Let $S$ be the set of primes $w$ of $L$ such that $E$ has bad reduction at $w_{K}$, $w_{K}$ is archimedean, or $w_{K}(n)>0$.

We now state the relevant conditions.

B1. The primes $v=(\pi)$ and $v^{\prime}=\left(\pi^{\prime}\right)$ are principal, with totally positive generators $\pi$ and $\pi^{\prime}$.

B2. Under the usual embedding, $E(K)$ lies in $n E\left(K_{v_{K}}\right)$.

B3. The generators $\pi$ and $\pi^{\prime}$ lie in $L_{w}^{\times n}$ for all $w \in S$.

B4. The order of the image of $\pi^{\prime}$ in $L_{v}^{\times} / L_{v}^{\times n}$ is $n$. Additionally, $\sigma \pi^{\prime}$ lies in $L_{v}^{\times n}$ for all nontrivial $\sigma \in \operatorname{Gal}(L / K)$.

B5. The primes $v_{K}, v_{K}^{\prime}$ split completely in $L$.

Lemma 4.1. There are infinitely many pairs of primes $(\pi),\left(\pi^{\prime}\right)$ satisfying conditions B1-B5.

Proof. Let $\mathfrak{m}$ be the modulus over $L$ given as the product of $n^{2}$ and all primes $w$ in $S$. Let $L_{\mathfrak{m}}$ be the ray class field of $L$ with modulus $\mathfrak{m}$. The modulus $\mathfrak{m}$ is rational over $K$, so that $L_{\mathfrak{m}}$ is Galois over $K$. Let $F$ be the compositum of $L_{\mathfrak{m}}, K\left([n]^{-1} E(K)\right)$, and the Hilbert class field of $K$. By the Chebotarev density theorem, there exists infinitely many primes $v_{K}$ of $K$ which split completely in $F$. Setting $v$ equal to any prime of $L$ lying over $v_{K}$, we use the same reasoning as in Lemma 3.1 to see that $v$ satisfies conditions B1-B5.

Choose any unit $\beta$ in $L_{v}$ which has order $n$ in $L_{v}^{\times} / L_{v}^{\times n}$. By the Chinese Remainder Theorem, there exists $\alpha \in L$ such that

$$
\begin{array}{ll}
\alpha \equiv \beta & (\bmod v) \\
\alpha \equiv 1 & (\bmod \sigma v) \quad \forall \sigma \neq 1 \in \operatorname{Gal}(L / K)
\end{array}
$$

Let $F^{\prime}$ be the ray class field for $L$ with modulus $\prod \sigma v$. The modulus is rational over $K$, so that $F^{\prime}$ is Galois over $K$. The Galois group $\operatorname{Gal}\left(F^{\prime} / L\right)$ is isomorphic to the class group with modulus $\prod \sigma v$ via the Artin reciprocity map. Let $\gamma_{L}$ in 
$\operatorname{Gal}\left(F^{\prime} / L\right)$ map to the class of $(\alpha)$ under this isomorphism. Then under the inclusion $\operatorname{Gal}\left(F^{\prime} / L\right) \hookrightarrow \operatorname{Gal}\left(F^{\prime} / K\right), \gamma_{L}$ maps to, say, $\gamma$. Let $[\gamma]$ be the conjugacy class of $\gamma$ in $\operatorname{Gal}\left(F^{\prime} / K\right)$.

One can find $\tau \in \operatorname{Gal}\left(F^{\prime} F / K\right)$ such that $\left.\tau\right|_{F^{\prime}}$ lies in $[\gamma]$, and $\left.\tau\right|_{F}$ is trivial. This follows because $F^{\prime} / L$ is ramified only at the $\sigma v$, while $F / L$ is unramified at those places; therefore $F \cap F^{\prime}$ is contained in the Hilbert class field of $L$. But $\gamma$ acts trivially on the Hilbert class field since $(\alpha)$ is principal. Therefore, $\tau$ as prescribed exists.

Now we apply the Chebotarev density theorem again to find $v_{K}^{\prime}$ corresponding to the conjugacy class of $\tau$. Choosing $v^{\prime}$ to be any place of $L$ lying over $v_{K}^{\prime}$, we use the same reasoning as before to conclude that $v^{\prime}$ satisfies the conditions.

4.2. The corestriction map. As before, choose a basis $(S, T)$ for $E[n]$ such that $e(S, T)=\zeta$. As before, our choice of basis yields an isomorphism

$$
\kappa: \mathrm{H}^{1}(L, E[n]) \rightarrow L^{\times} / L^{\times n} \times L^{\times} / L^{\times n}
$$

Let $\pi, \pi^{\prime}$ be as in the previous section, and choose $\xi$ so that $\kappa(\xi)=\left(\pi, \pi^{\prime n / \ell}\right)$. Let cores : $\mathrm{H}^{1}(L, E[n]) \rightarrow \mathrm{H}^{1}(K, E[n])$ be the corestriction map, and $\eta=\operatorname{cores}(\xi)$. We wish to show that the curve corresponding to $\eta$ satisfies the conditions of our theorem. But it is too difficult to compute the obstruction map $\mathrm{Ob}(\eta)$, so we will instead use $\mathrm{Ob}(\operatorname{res} \eta)$, where res is the restriction map

$$
\mathrm{H}^{1}(K, E[n]) \rightarrow \mathrm{H}^{1}(L, E[n]) .
$$

Therefore, we need to compute reso $\operatorname{cores}(\xi)$. Note that cores o res is well-known and equal to $[L: K]$, but the same is not true of res o cores.

For any $G_{K}$-module $M$ and nonnegative integer $r$, one has an action of $\operatorname{Gal}(L / K)$ on $\mathrm{H}^{r}(L, M)$. This action is induced by the action on homogeneous $r$-cochains

$$
c^{\sigma}\left(\gamma_{1}, \ldots, \gamma_{r}\right)=\sigma c\left(\sigma^{-1} \gamma_{1} \sigma, \ldots, \sigma^{-1} \gamma_{r} \sigma\right)
$$

where $\sigma \in \operatorname{Gal}(L / K)$ and $\gamma_{i} \in G_{L}$. (Actually, we must lift $\sigma$ to any fixed element of $G_{K}$ when acting by conjugation on the $\gamma_{i}$.) Define $\mathrm{Nm}: \mathrm{H}^{r}(L, M) \rightarrow \mathrm{H}^{r}(L, M)$ by

$$
\operatorname{Nm}(\theta)=\sum_{\sigma \in \operatorname{Gal}(L / K)} \theta^{\sigma} .
$$

Lemma 4.2. For $\theta \in \mathrm{H}^{r}(L, M)$, res $\circ \operatorname{cores} \theta=\mathrm{Nm} \theta$, where res and cores are the obvious restriction and corestriction maps.

Proof. This follows from the definition of corestriction and dimension shifting; see [17, p. 119].

Therefore $\operatorname{cores} \eta=\operatorname{Nm} \xi$. Unfortunately, $\kappa$ is not $\operatorname{Gal}(L / K)$-equivariant, so that $\kappa(\mathrm{Nm} \xi) \neq\left(\mathrm{Nm} \pi, \mathrm{Nm} \pi^{\prime n / \ell}\right)$. Instead, we have a twisted norm on $L^{\times} / L^{\times n} \times$ $L^{\times} / L^{\times n}$, which we now describe. Let

$$
\begin{aligned}
\operatorname{Gal}(L / K) & \rightarrow \mathrm{GL}_{2}(\mathbb{Z} / n \mathbb{Z}) \\
\sigma & \mapsto M_{\sigma}
\end{aligned}
$$

be the representation of $\operatorname{Gal}(L / K)$ on $E[n]$ with respect to the basis $(S, T)$. The determinant of $M_{\sigma}$ is given by the $n$th cyclotomic character. 
Proposition 4.3. Suppose $\sigma \in \operatorname{Gal}(L / K), \xi \in \mathrm{H}^{1}(L, E[n])$ and $\kappa(\xi)=(a, b)$. Then

$$
\kappa\left(\xi^{\sigma}\right)=\frac{M_{\sigma}}{\operatorname{det} M_{\sigma}}(\sigma a, \sigma b)
$$

where for $M \in \mathrm{GL}_{2}(\mathbb{Z} / n \mathbb{Z})$, if

$$
M=\left[\begin{array}{cc}
i & j \\
k & l
\end{array}\right]
$$

then $M(a, b)=\left(a^{i} b^{j}, a^{k} b^{l}\right)$.

Proof. Let $\rho: E[n] \rightarrow \mu_{n} \times \mu_{n}$ be the isomorphism given by the basis $(S, T)$. Write $\left(\mu_{n} \times \mu_{n}\right)_{\rho}$ for the $\operatorname{Gal}(L / K)$-module with underlying group $\mu_{n} \times \mu_{n}$, but with module-structure making $\rho$ an isomorphism of Galois modules; that is

$$
\begin{aligned}
\sigma\left(\zeta_{1}, \zeta_{2}\right)_{\rho} & =\rho \sigma \rho^{-1}\left(\zeta_{1}, \zeta_{2}\right) \\
& =M_{\sigma}\left(\zeta_{1}, \zeta_{2}\right) .
\end{aligned}
$$

Consider the diagram

$$
\begin{aligned}
L^{\times} / L^{\times n} \times L^{\times} / L^{\times n} \stackrel{\psi}{\longrightarrow} & \mathrm{H}^{1}\left(L, \mu_{n} \times \mu_{n}\right) \\
& \downarrow^{i_{*}} \\
& \mathrm{H}^{1}\left(L,\left(\mu_{n} \times \mu_{n}\right)_{\rho}\right) \underset{\varphi}{\longrightarrow} \mathrm{H}^{1}(L, E[n])
\end{aligned}
$$

The isomorphism $\psi$ comes from the usual Kummer isomorphism. The map $i_{*}$ is induced by the canonical isomorphism of the underlying groups $i: \mu_{n} \times \mu_{n} \rightarrow$ $\left(\mu_{n} \times \mu_{n}\right)_{\rho}$. The map $\varphi$ is induced by the map sending $(\zeta, 1)$ to $S$ and $(1, \zeta)$ to $T$ (compare to $\left.\kappa^{-1}\right)$. Then the horizontal arrows are both $\operatorname{Gal}(L / K)$-equivariant, and the composition $\varphi i_{*} \psi$ is equal to $\kappa^{-1}$.

Let $\gamma \in \operatorname{Gal}(\bar{L} / L)$ and lift $\sigma$ arbitrarily to an element, also written $\sigma$, of $\operatorname{Gal}(\bar{K} / K)$. We have

$$
\begin{aligned}
{\left[i_{*} \psi(a, b)\right]^{\sigma}(\gamma) } & =\sigma i\left(\psi(a, b)\left(\sigma^{-1} \gamma \sigma\right)\right) \\
& =\sigma i\left(\sigma^{-1} \sigma \psi(a, b)\left(\sigma^{-1} \gamma \sigma\right)\right) \\
& =\sigma i\left(\sigma^{-1} \psi(a, b)^{\sigma}(\gamma)\right) \\
& =\sigma i\left(\sigma^{-1}[\psi(\sigma a, \sigma b)(\gamma)]\right) \\
& =\frac{M_{\sigma}}{\operatorname{det} M_{\sigma}} i[\psi(\sigma a, \sigma b)(\gamma)] \\
& =i_{*} \psi\left(\frac{M_{\sigma}}{\operatorname{det} M_{\sigma}}(\sigma a, \sigma b)\right)(\gamma)
\end{aligned}
$$

The equality 4.5 follows by the definition of the $\operatorname{Gal}(L / K)$-action on $\mathrm{H}^{1}\left(L, \mu_{n} \times\right.$ $\left.\mu_{n}\right)$. Since $\psi$ is a $\operatorname{Gal}(L / K)$-morphism, we obtain (4.6). Finally, (4.7) follows from the action of $\sigma$ on $E[n]$ by the matrix $M_{\sigma}$, and the action of $\sigma^{-1}$ on $\mu_{n} \times \mu_{n}$ by $\left(\operatorname{det} M_{\sigma}\right)^{-1}$.

Now apply $\varphi$ to both sides of the equation to obtain

$$
\left[\kappa^{-1}(a, b)\right]^{\sigma}=\kappa^{-1}\left(\frac{M_{\sigma}}{\operatorname{det} M_{\sigma}}(\sigma a, \sigma b)\right)
$$

from which the lemma follows. 
Corollary 4.4. Let $\kappa(\xi)=(a, b)$. Then $\kappa(\mathrm{Nm} \xi)$ equals

$$
\left(\prod\left(\operatorname{det} M_{\sigma}\right)^{-1} \sigma a^{i(\sigma)} \sigma b^{j(\sigma)}, \prod\left(\operatorname{det} M_{\sigma}\right)^{-1} \sigma a^{k(\sigma)} \sigma b^{\ell(\sigma)}\right)
$$

where the product is taken over $\sigma \in \operatorname{Gal}(L / K)$.

4.3. Computation of the obstruction map. Let $\xi \in \mathrm{H}^{1}(L, E[n])$ satisfy $\kappa(\xi)=$ $\left(\pi, \pi^{\prime n / \ell}\right)$, where $\pi$ and $\pi^{\prime}$ were chosen as in Lemma 4.1. Let $\eta=\operatorname{cores} \xi$. In order to compute $\mathrm{Ob}(\eta)$, we instead compute res $\mathrm{Ob}(\eta)=\mathrm{Ob}(\operatorname{res} \eta)$, or $\mathrm{Ob}(\mathrm{Nm} \xi)$. But the $n$-torsion $E[n]$ is rational over $L$, so by Proposition 2.4 we may use the Hilbert symbol to compute $\mathrm{Ob}(\mathrm{Nm} \xi)$.

To ease this computation, we will first do the case $\ell=1$. Equivalently, let $\xi_{0} \in \mathrm{H}^{1}(L, E[n])$ satisfy $\kappa\left(\xi_{0}\right)=(\pi, 1)$ and let $\eta_{0}=\operatorname{cores} \xi_{0}$. Let

$$
\begin{aligned}
& c=\prod\left(\operatorname{det} M_{\sigma}\right)^{-1} \sigma \pi^{i(\sigma)} \\
& d=\prod\left(\operatorname{det} M_{\sigma}\right)^{-1} \sigma \pi^{k(\sigma)} .
\end{aligned}
$$

Thus, $\operatorname{Nm} \kappa^{-1}(\pi, 1)=\kappa^{-1}(c, d)$.

Lemma 4.5. Let $w$ be any place of $L$ satisfying $w(\sigma \pi)=0$ for all $\sigma$. Then the local Hilbert symbol $\langle c, d\rangle_{w}$ is trivial.

Proof. If $w$ is non-archimedean and $w(n)=0$, then $c$ and $d$ are both units in $L_{w}$. Therefore by Lemma 2.5 the Hilbert symbol is trivial.

If $w(n) \neq 0$, then $w$ lies in $S$. According to condition B3, $\pi$ and its conjugates lie in $L_{w}^{\times n}$. Again, the Hilbert symbol is trivial.

Finally, condition B1 guarantees that the Hilbert symbol at all the archimedean places is automatically trivial.

Lemma 4.6. $\mathrm{Ob} \eta_{0}=0$.

Proof. We compute the class of $\mathrm{Ob} \eta_{0}$ at each place of $K$. The corestriction map on $\mathrm{H}^{1}(L, E[n])$ induces a homomorphism

$$
\oplus \operatorname{cores}_{w}: \oplus \mathrm{H}^{1}\left(L_{w}, E[n]\right) \rightarrow \mathrm{H}^{1}\left(K_{w_{K}}, E[n]\right)
$$

where the sum is over all places $w$ of $L$ lying over a given place $w_{K}$ of $K$. Recall that $\eta_{0}=\operatorname{cores} \xi_{0}$. By construction, $\xi_{0}$ is locally trivial everywhere except at $v$ and its conjugates. Triviality at $w_{K} \neq v_{K}$ follows.

Since the local invariants of a global Brauer class sum to zero, the obstruction map at $v_{K}$ must also be zero.

Note that the above proves the $\ell=1$ case of Theorem 1.2 .

We now consider $\xi$ and $\eta=\operatorname{cores} \xi$. Let

$$
\begin{aligned}
& c^{\prime}=\prod\left(\operatorname{det} M_{\sigma}\right)^{-1} \sigma \pi^{\prime \frac{n}{\ell} j(\sigma)} \\
& d^{\prime}=\prod\left(\operatorname{det} M_{\sigma}\right)^{-1} \sigma \pi^{\prime \frac{n}{\ell} l(\sigma)} .
\end{aligned}
$$

We have $\operatorname{Nm} \kappa^{-1}\left(1, \pi^{\prime n / \ell}\right)=\kappa^{-1}\left(c^{\prime}, d^{\prime}\right)$, and $\kappa(\mathrm{Nm} \xi)=\left(c c^{\prime}, d d^{\prime}\right)$. We wish to compute the Hilbert symbol $\left\langle c c^{\prime}, d d^{\prime}\right\rangle$.

Lemma 4.7. Let $w$ be any place of $L$ satisfying $w(\sigma \pi)=w\left(\sigma \pi^{\prime}\right)=0$ for all $\sigma$. Then the local Hilbert symbol $\left\langle c c^{\prime}, d d^{\prime}\right\rangle_{w}$ is trivial.

Proof. The argument is identical to the proof of Lemma 4.5 . 
Lemma 4.8. Let $v$ be the place of $L$ corresponding to $\pi$. Then $\left\langle c c^{\prime}, d d^{\prime}\right\rangle_{v}$ has exact order $\ell$.

Proof. By bilinearity, we have

$$
\left\langle c c^{\prime}, d d^{\prime}\right\rangle=\langle c, d\rangle+\left\langle c, d^{\prime}\right\rangle+\left\langle c^{\prime}, d\right\rangle+\left\langle d, d^{\prime}\right\rangle .
$$

Since $c^{\prime}, d, d^{\prime}$ are all units in $L_{v}^{\times}$, the last two terms are zero by Lemma 2.5 As for $\langle c, d\rangle$, this equals $\mathrm{Ob}\left(\mathrm{res} \eta_{0}\right)$, which is zero by Proposition 2.7 and Lemma 4.6 .

Now $c=u \pi$, where $u$ is some unit in $L_{v}^{\times}$, so that $\left\langle c, d^{\prime}\right\rangle_{v}=\left\langle\pi, d^{\prime}\right\rangle_{v}$. By condition $\mathrm{B} 4, d^{\prime} \equiv \pi^{\prime n / \ell}\left(\bmod L^{\times n}\right)$, so that $\left\langle c, d^{\prime}\right\rangle_{v}=\left\langle\pi, \pi^{\prime n / \ell}\right\rangle_{v}$. Again applying condition B4 and Lemma 2.5, we see that $\left\langle c, d^{\prime}\right\rangle_{v}$ has order $\ell$.

Let $v^{\prime}$ be the place of $L$ corresponding to $\pi^{\prime}$.

Proposition 4.9. Ob $\eta$ has order $\ell$ at $v_{K}$ and $v_{K}^{\prime}$, and is trivial at all other places.

Proof. The proof of triviality at $w_{K} \neq v_{K}, v_{K}^{\prime}$ is identical to the first part of the proof of Lemma 4.6

Since the local invariants of a global Brauer class sum to zero, it suffices to show that $(\mathrm{Ob} \eta)_{v_{K}}$ has order $\ell$. The diagram

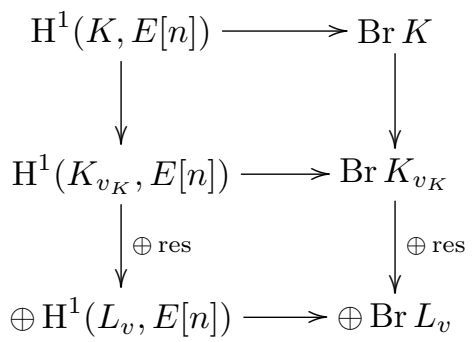

commutes. The horizontal maps are the relevant obstruction maps. Commutativity follows from functoriality of localization and restriction in nonabelian cohomology. But $v_{K}$ splits completely in $L$ by condition B5, so that $K_{v_{K}} \cong L_{v}$. Thus if we consider the restriction map onto a single factor

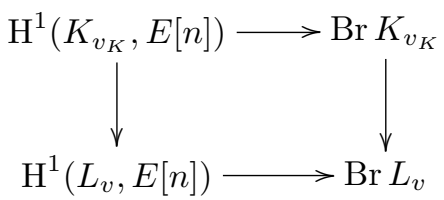

then the vertical maps are isomorphism. Therefore the order of $(\mathrm{Ob} \eta)_{v_{K}}$ equals the order of $(\mathrm{Ob} \text { res } \eta)_{v}$, or rather $(\mathrm{ObNm} \xi)_{v}$.

Since $E[n] \subset E(L) \subset E\left(L_{v}\right)$, we may use the Hilbert symbol to evaluate the obstruction map. By construction, $\kappa(\mathrm{Nm} \xi)=\left(c c^{\prime}, d d^{\prime}\right)$. The result follows from Lemma 4.8

4.4. End of proof. Let $X$ be the genus 1 curve over $K$ represented by the class $\eta$ in $\mathrm{H}^{1}(K, E[n])$. Clearly the period of $X$ divides $n$. Suppose that the period is smaller. Then there is some positive integer $m$ with $m<n$ and some $x \in E(K)$ such that

$$
\delta x+m \eta=0 .
$$


In particular, this holds locally at $v_{K}$. But by condition $\mathrm{B} 2, \delta x$ is trivial at $v_{K}$ for all $x \in E(K)$. Therefore we must have $m \eta=0$ at $v_{K}$. In particular, this must hold when we restrict to $L_{v}$. But this cannot be, for

$$
\begin{aligned}
\kappa\left(\operatorname{res}_{L / K} m \eta\right) & =m \kappa(\operatorname{res} \eta) \\
& =m\left(u_{1} \cdot \pi, u_{2}\right) \\
& =\left(u_{1}^{m} \cdot \pi^{m}, u_{2}^{m}\right)
\end{aligned}
$$

for some $u_{1}, u_{2}$ which are units in $L_{v}$. We conclude that $X$ has exact period $n$.

By Proposition 4.9, Ob $\eta$ has order $\ell$. Applying Proposition 2.2 we see that the index of $X$ divides $n \ell$. Suppose the index is $n \ell^{\prime}$, with $\ell^{\prime}<\ell$. By Proposition 2.2 there is a class $\eta^{\prime}$ in $\mathrm{H}^{1}\left(K, E\left[n \ell^{\prime}\right]\right)$ representing $X$ such that $\mathrm{Ob}_{n \ell^{\prime}} \eta^{\prime}=0$. Let $j_{*}$ be the canonical homomorphism

$$
\mathrm{H}^{1}(K, E[n]) \rightarrow \mathrm{H}^{1}\left(K, E\left[n \ell^{\prime}\right]\right) .
$$

Since $\eta$ also represents $X$, there exists $x \in E(K)$ such that

$$
\eta^{\prime}=j_{*}(\eta)+\delta x
$$

By Proposition 2.3 ,

$$
\mathrm{Ob}_{n \ell^{\prime}} \eta^{\prime}=\mathrm{Ob}_{n \ell^{\prime}} j_{*}(\eta)+T(x, X)
$$

where $T$ is the Tate pairing. Consider the latter equation locally at $v_{K}$. By condition B2, $x$ lies in $n E\left(K_{v_{K}}\right)$, so the Tate pairing is zero. The left hand term is zero by hypothesis. But by Proposition 2.7. $\mathrm{Ob}_{n \ell^{\prime}} j_{*}(\eta)=\ell^{\prime} \mathrm{Ob}_{n} \eta$, and the latter is not zero since $\mathrm{Ob}_{n} \eta$ has order $\ell$ and $\ell^{\prime}<\ell$. This yields a contradiction. Therefore the index of $X$ must be exactly $n \ell$.

\section{EVEN PERIOD}

Assume now that $n$ is a power of 2 . There is a single problem with the above arguments: the obstruction map need not equal the Hilbert symbol; instead, according to Proposition 2.4. $\mathrm{Ob}(\xi)-\langle\kappa(\xi)\rangle$ is killed by 2 .

Suppose we undertake our construction anyway, yielding $\eta \in \mathrm{H}^{1}(K, E[n])$. The two problems above imply that our calculation of $\mathrm{Ob}(\eta)$ may be off by an element of $(\mathrm{Br} K)[2]$. If $\ell \geq 4$, then the difference is immaterial, and the proof works. We now consider the cases $\ell=1$ and $\ell=2$.

Assume that $\ell=1$. Using the construction from the previous section, we can come up with a curve $X^{\prime}$ with period $2 n$ and index either $2 n$ or $4 n$, though we are not able to determine which of the two holds. Suppose $\eta \in \mathrm{H}^{1}(K, E[2 n])$ represents $X^{\prime}$ with $\mathrm{Ob}_{2 n}(\eta) \in(\operatorname{Br} K)[2]$. Let $X$ be the curve with class $2 \eta$. Note that we may view $2 \eta$ as an element of $\mathrm{H}^{1}(K, E[n])$; in particular, $X$ has period $n$. To show that $X$ has index $n$, it suffices to show that $\mathrm{Ob}_{n}(2 \eta)=0$. But by Proposition 2.7. $\mathrm{Ob}_{n}(2 \eta)=2 \mathrm{Ob}_{2 n} \eta=0$.

The procedure for $\ell=2$ is similar: construct $X^{\prime}$ as before with period $2 n$ and index $8 n$, so that $\mathrm{Ob}_{2 n}(\eta)$ has order 4 . Then $\mathrm{Ob}_{n}(2 \eta)$ has order 2, and the curve $X$ represented by $2 \eta$ has period $n$ and index $2 n$.

\section{FinAl REMARKS}

The results of the paper should generalize to function fields, that is, finite extensions of $\mathbb{F}_{p}(T)$. The only wrinkle occurs when $p \mid n$, for then $E[n]$ is no longer an étale group scheme. Clark, in a personal communication has results making use of 
a so-called flat Hilbert symbol. If the obstruction map can be controlled with the flat symbol, then hopefully Theorem 1.2 can be extended.

The description of the $\operatorname{Gal}(L / K)$ action on $\mathrm{H}^{1}(L, E[n])$ yields an explicit description, in most cases, of $\mathrm{H}^{1}(K, E[n])$ for $K$ an arbitrary number field, in the following manner. Given $E / K$, for $n$ not divisible by a finite set of primes, we know that $\operatorname{Gal}(L / K)$ surjects onto $\operatorname{Aut}(E[n])$. One can show that $\mathrm{H}^{q}(L / K, E[n])=0$ for all $q$, whence the inflation-restriction sequence yields an isomorphism

$$
\mathrm{H}^{1}(K, E[n])=\mathrm{H}^{1}(L, E[n])^{\operatorname{Gal}(L / K)} .
$$

From Proposition 4.3 , one obtains the desired description of $\mathrm{H}^{1}(K, E[n])$ as pairs of elements of $L^{\times} / L^{\times n}$.

Finally, a consequence of Lemma 4.6 is a nontrivial relation on the Hilbert symbols of an element $\pi$ and its conjugates; namely

$$
\sum \frac{k(\sigma)}{\operatorname{det} M_{\sigma}}\langle\sigma \pi, \pi\rangle=0
$$

where $k(\sigma)$ comes from the Galois representation on the torsion, and $\operatorname{det} M_{\sigma}$ is identical to the cyclotomic character. No other relation of this type is known to the author.

\section{REFERENCES}

[1] J. Cassels, Arithmetic on curves of genus 1. V. Two counterexamples, J. London Math. Soc. 41 (1966), 244-248.

[2] Pete Clark, The period-index problem in WC-groups I: elliptic curves, J. Number Theory 114 (2005), 193-208.

[3] - There are genus one curves of every index over every number field, J. Reine Angew. Math. 594 (2006), 201-206.

[4] Pete Clark, On the indices of curves over local fields, Manuscripta Math. 124 (2007), no. 4, 411-426. MR MR2357791 (2008m:11121)

[5] Pete Clark and Shahed Sharif, Period, index, and the Tate-Shafarevich group of elliptic curves, Submitted for publication.

[6] Cristian González-Avilés, Brauer groups and Tate-Shafarevich groups, J. Math. Sci. Univ. Tokyo 10 (2003), 391-419.

[7] Alexander Grothendieck, Le groupe de Brauer. III. Exemples et compléments, Dix Exposés sur la Cohomologie des Schémas, North-Holland, Amsterdam, 1968, pp. 88-188. MR 39 \#5586c

[8] Serge Lang and John Tate, Principal homogeneous spaces over abelian varieties, Amer. J. Math. 80 (1958), 659-684.

[9] Stephen Lichtenbaum, The period-index problem for elliptic curves, Amer. J. of Math. 90 (1968), 1209-1223.

[10] _ Duality theorems for curves over p-adic fields, Invent. math. 7 (1969), 120-136.

[11] Qing Liu, Dino Lorenzini, and Michel Raynaud, Néron models, Lie algebras, and reduction of curves of genus one, Invent. Math. 157 (2004), 455-518.

[12] _ On the Brauer group of a surface, Invent. Math. 159 (2005), no. 3, 673-676.

[13] David Mumford, On the equations defining abelian varieties. I, Invent. Math. 1 (1966), 287354. MR 0204427 (34 \#4269)

[14] Catherine O'Neil, The period-index obstruction for elliptic curves, J. Number Theory 95 (2002), 329-339.

[15] _ Erratum to the period-index obstruction for elliptic curves, J. Number Theory 109 (2004), 390.

[16] Bjorn Poonen and Michael Stoll, The Cassels-Tate pairing on polarized abelian varieties, Ann. of Math. (2) 150 (1999), no. 3, 1109-1149. MR 2000m:11048

[17] Jean-Pierre Serre, Local fields, Springer-Verlag, New York, 1979, Translated from the French by Marvin Jay Greenberg. MR 82e:12016 
[18] Shahed Sharif, Curves with prescribed period and index over local fields, J. Algebra 314 (2007), no. 1, 157-167.

[19] Joseph H. Silverman, The arithmetic of elliptic curves, Springer-Verlag, New York, 1992, Corrected reprint of the 1986 original. MR 95m:11054

[20] William Stein, There are genus one curves over $\mathbb{Q}$ of every odd index, J. Reine Angew. Math. 547 (2002), 139-147.

[21] Jakob Stix, On the period-index problem in light of the section conjecture, arXiv:mathnt/0802.4125v1, 2008.

[22] Yuri Zarhin, Noncommutative cohomology and Mumford groups, Mat. Zametki 15 (1974), 415-419.

Dept. of Mathematics, Duke University, Durham, NC 27708

E-mail address: sharif@math.duke.edu 